\title{
MODELING OF PHOTOELASTIC CONSTANT DEPENDENT ON ARBITRARY CRYSTAL ORIENTATION
}

\author{
Lubna Jahan Rashid Pinky ${ }^{(1)}$ and Abu Naim Rakib Ahmed ${ }^{(2)}$
}

1. Lecturer, Electrical Electronic and Engineering Department, IUBAT, Dhaka

2. Lecturer, Electrical Electronic and Engineering Department, Khulna University of Engineering \& Technology

\begin{abstract}
A mathematical model is proposed to determine the photoelastic constants in arbitrary crystal orientation of Zincblende crystal structure. Tensor rotation technique is applied using Euler's rotation theorem to develop the model. The model is applicable to evaluate photoelastic constants in terms of P11-P12 and P44 in any crystal plane by controlling the rotation angle using the model. P11-P12 and P44 are calculated for Silicon crystal and found that the values of these constants are strongly dependent on crystal orientations. The outcome of this research enables us to evaluate quantitative amount of strain in polycrystalline silicon material (solar cell material) using Scanning Infrared Polariscope.
\end{abstract}

KEY WORDS - Photoelastic constant, polycrystalline silicon, tensor rotation, crystal orientation, scanning infrared Polariscope method.

\subsection{INTRODUCTION}

The application of an external stress to solid produces a change in crystal symmetry and lattice parameters, which results in significant change in optical properties. By the application of external stress an optically isotropic semiconductor becomes birefringent. This phenomenon is known as photoelasticity. Photoelastic constant is very important to determine the strain in semiconductor devices using the experimental technique known as Scanning Infrared Polariscope. In this technique the absolute values of the residual strain components: $\left|S_{y y}-S_{z z}\right|$ and $2\left|S_{y z}\right|$, which are the difference of the tensile strains along the radial and tangential directions in a cylindrical coordinate system, that along the $\mathrm{y}$ and $\mathrm{x}$ axes in the crystallographic Cartesian coordinate system and the shear strain between the $\mathrm{y}$ and $\mathrm{z}$ axis can be quantitatively characterized by knowing the photoelastic constants. The relationship with the strain components and photoelastic constants for the method is ${ }^{[1]}$

$$
\begin{aligned}
& \left|S_{y y}-S_{z z}\right|=k \delta\left|\frac{\cos 2 \phi}{p_{11}-p_{12}}\right| \\
& 2\left|S_{y z}\right|=k \delta\left|\frac{\sin 2 \phi}{p_{44}}\right|
\end{aligned}
$$

Where, $\mathrm{k}=\frac{\lambda}{\pi d n_{0}{ }^{3}}$

$$
\begin{aligned}
& P_{i j}=\text { Photo elastic constant } \\
& n_{0}=\text { the refractive index }
\end{aligned}
$$

$\left|S_{y y}-S_{z z}\right|=$ the difference of the tensile strains along the crystallographic $y$ and $z$ axes $2\left|S_{y z}\right|=$ the shear strain between the $y$ and $\mathrm{z}$ axes Photoelastic constant depends on the crystalline orientations. Crystal orientation changes randomly in polycrystalline material. Polycrystalline silicon is a key component of solar panel construction. Monocrystalline silicon is higher priced and more efficient than polycrystalline. But low cost solar cell can be fabricated by polycrystalline silicon though its performance is very poor which is assumed to be due to the strain induced in the material during the growth and cooling processes. Since the strain in semiconductor material is highly dependent in crystal orientations, to characterize the strain in solar cell material by the Scanning Infrared Polariscope, it is very much important to know the photoelastic constant in different crystal orientations. Photoelastic constant can only be determined experimentally in (100) and (111) crystal orientations. But to characterize the quantitative amount of strain in polycrystalline silicon-based solar cell, it is needed to know the photoelastic constants in arbitrary crystal direction. In this work, a mathematical model is proposed to determine photoelastic constants in arbitrary crystal orientation using tensor rotation technique. Finally, quantitative amount of photoelastic constants in terms of $P_{11}^{\prime}-P_{12}^{\prime}$ and $P_{44}^{\prime}$ are calculated in different orientations for $\mathrm{Si}$ crystal as an example. However, the formulations can be applied to evaluate photoelastic constants in any Zincblende crystal structure. 


\subsection{Proposed System ModeL}

For the determination of photoelastic constants in arbitrary crystal orientation two system models are proposed.

\subsection{SYSTEM MODEL 1}

To determine photoelastic constant in any direction at $\mathrm{XY}$ plane or in any plane from $\mathrm{XY}$ to $X^{\prime} Z^{\prime}$ plane, we have developed a system model based on the relationship of the general (hkl) coordinate system to the conventional (001) crystal coordinate system. At first the $\mathrm{X}$ axis is rotated in $\mathrm{XY}$ plane keeping $\mathrm{Z}$ axis constant by an angle $\phi$ and so the $\mathrm{Y}$ axis is also rotated by the same angle, $\phi$. After the first rotation, the new positions of $\mathrm{X}$ axis and $\mathrm{Y}$ axis are denoted as $X^{\prime}$ and $Y^{\prime}$. Again the $X^{\prime}$ axis is rotated by an angle $\theta$ about the $Y^{\prime}$ axis and so the $Z^{\prime}$ axis is also rotated by the same angle $\theta$. The final position of $X$ " and $Z^{\prime \prime}$ is obtained after the second rotation.

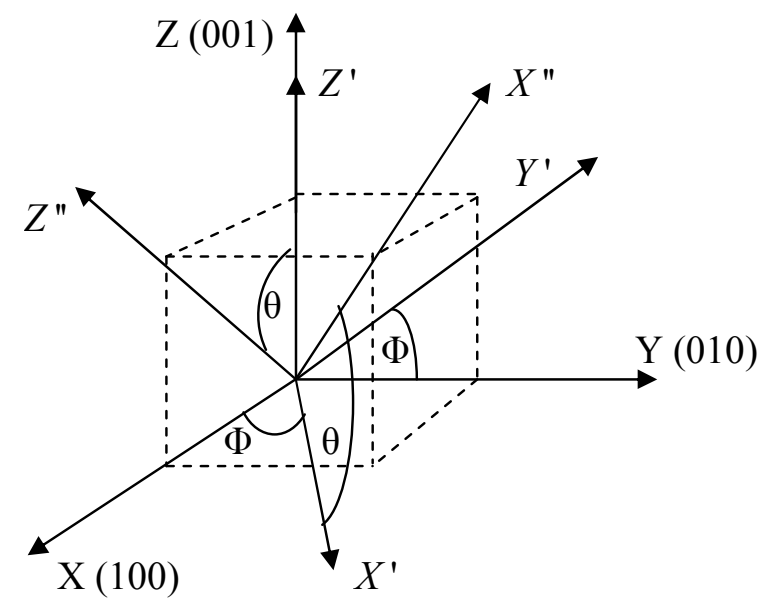

Fig.1: Relationship of the general (hkl) coordinate system to the conventional ( 001$)$ crystal coordinate system-

The angles, $\phi$ and $\theta$ of the (hkl) direction relative to a coordinate system fixed onto the (100), (010) and (001) axes, can be given by the following relationships [2]

$$
\begin{aligned}
& \tan \varphi=\frac{k}{h} \\
& \tan \theta=\frac{\sqrt{h^{2}+k^{2}}}{l}
\end{aligned}
$$

Where, the indices $\mathrm{h}, \mathrm{k}$, and $\mathrm{l}$ are the real integers for the case when the crystalline surface is specified in terms of the azimuthally angle, $\phi$ and polar angle $\theta$.

\subsection{SySTEM MOdEL 2}

Since the above model can not be applied to determine photoelastic constants in any crystal plane between (100) and (011), a new system model is developed which is based on the relationship among the angles $\phi, \theta$ and $\alpha$.

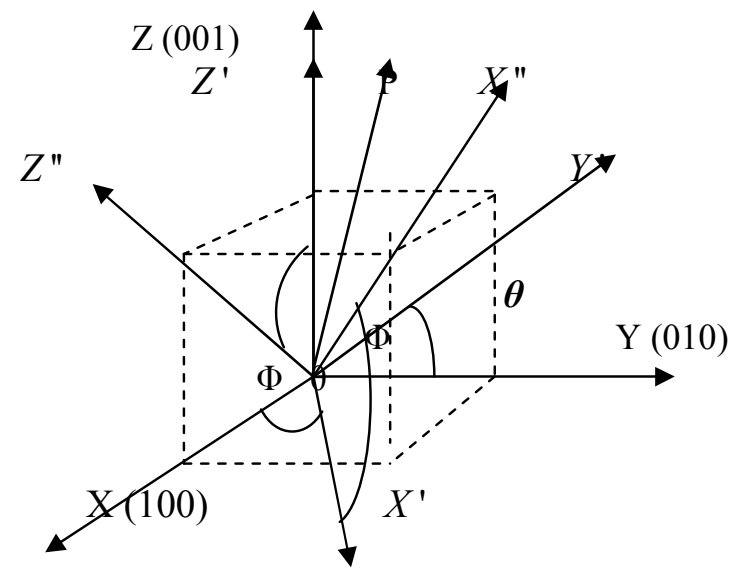

Fig.2: System model for evaluating photoelastic constant in some special orientations

Consider a unit vector $\mathrm{P}$ is moving from (100) direction to (011) direction. For a particular position of $\mathrm{P}$, it makes an angle $\alpha$ with $\mathrm{X}$ axis. Let any point in $\mathrm{XY}$ plane is $(x, x \tan \phi, 0)$ after rotating by an angle $\Phi$ and then the same point rotates from $X^{\prime} Z^{\prime}$ plane by an angle $\theta$ about $Y^{\prime}$ axis. So the final position is now $(x, x \tan \phi, x \tan \phi)$. The direction cosine ratio between these two points is

$$
\cos \theta=\frac{\sec \phi}{\sqrt{\sec ^{2} \phi+\tan ^{2} \phi}}
$$

If we express $\cos \theta$ in terms of $\tan \theta$ then it can be written as

$$
\tan \theta=\sin \phi
$$

The position of $\mathrm{P}$ meets in the same point which was obtained by the angles $\Phi$ and $\theta$ rotation. So the direction cosine between the point at $\mathrm{X}$ axis $(\mathrm{x}, 0,0)$ and the point $(x, x \tan \phi, x \tan \phi)$ is

$$
\cos \alpha=\frac{1}{\sqrt{\sec ^{2} \phi+\tan ^{2} \phi}}
$$

So the relation among the angles $\phi, \theta$ and $\alpha$ can be found by the equations (6) and (7) which is

$$
\cos \alpha=\cos \phi \cos \theta
$$




\subsection{MATHEMATiCAL AnALYSIS}

Here we have developed a mathematical model based on tensor rotation technique to determine photoelastic constants in arbitrary crystal orientation.

\subsection{Mathematical Analysis for System MODEL1}

From the tensor rotation technique, the rotation matrixes for the first and second type rotation shown in figure (1) are respectively

$$
C=\left[\begin{array}{ccc}
\cos \phi & \sin \phi & 0 \\
-\sin \phi & \cos \phi & 0 \\
0 & 0 & 1
\end{array}\right], B=\left[\begin{array}{ccc}
\cos \theta & 0 & -\sin \theta \\
0 & 1 & 0 \\
\sin \theta & 0 & \cos \theta
\end{array}\right]
$$

According to Euler's rotation theorem, the general rotation matrix $\mathrm{M}$ for two types of tensor rotation can be written as ${ }^{[3]}$,

$$
\begin{aligned}
& \mathrm{M}=\mathrm{BC} \\
& =\left[\begin{array}{ccc}
\cos \theta \cos \phi & \cos \theta \sin \phi & -\sin \theta \\
-\sin \phi & \cos \phi & 0 \\
\sin \theta \cos \phi & \sin \theta \sin \phi & \cos \theta
\end{array}\right]
\end{aligned}
$$

The relation between photo elastic constant, $P_{i j k l}$, stress, $X_{i j}$ and change in refractive index, $\Delta n$ in the semiconductor material is ${ }^{[4]}$

$$
\Delta n=-P_{i j k l} X_{i j}
$$

When equation (10) is expanded, it yields $81 P_{i j k l}$ components. According to tensor rotation rule, symmetry transformation results in equivalent 36 components form those 81 photoelastic constant ${ }^{[4]}$. Again by employing an additional Symmetry element for the cubic system we can have further reduced set of three independents components.

If photoelastic constant in any direction is known, its value in other direction can be calculated using Tensor rotation rule which is ${ }^{[4]}$

$$
P_{\text {mnop }}^{\prime}=\sum_{i, j, k, l=1}^{3} a_{m i} a_{n j} a_{o k} a_{p l} P_{i j k l}
$$

Where, $a_{m i}, a_{n j}, a_{o k}, a_{p l}$ are the respective direction cosines between the two sets of coordinate axis before and after symmetry transformation, respectively, in the case of Cartesian tensors which are expressed in the form of rotation matrix shown in equation (9). Pijkl is the value of known photo elastic constant and $P_{m n o p}^{\prime}$ indicates the value which is to be evaluated. By expanding equation (11) using the independent components of photoelastic constant we get

$P_{11}^{\prime}=P_{11}\left(\cos ^{4} \theta \cos ^{4} \Phi+\cos ^{4} \theta \sin ^{4} \Phi+\sin ^{4} \theta\right)+2$ $P_{12}\left(\cos ^{4} \theta \cos ^{2} \Phi \sin ^{2} \Phi+\cos ^{2} \theta \cos ^{2} \Phi \sin ^{2} \theta+\cos ^{2} \theta\right.$ $\left.\sin ^{2} \Phi \sin ^{2} \theta\right)+P_{44} \quad\left(\cos ^{4} \theta \cos ^{2} \Phi \sin ^{2} \Phi+\right.$ $\left.\cos ^{2} \theta \sin ^{2} \Phi \sin ^{2} \theta+\cos ^{2} \theta \cos ^{2} \Phi \sin ^{2} \theta\right)$ (12)

$P_{12}^{\prime}=P_{11}\left(\cos ^{2} \theta \cos ^{2} \Phi \sin ^{2} \Phi+\cos ^{2} \theta \sin ^{2} \Phi \cos ^{2} \Phi\right)$

$+P_{12}\left(\cos ^{2} \theta \cos ^{4} \phi+\cos ^{2} \theta \sin ^{4} \Phi+\sin ^{2} \theta \sin ^{2} \Phi\right.$ $\left.+\sin ^{2} \theta \cos ^{2} \Phi\right)+P_{44}\left(-\cos ^{2} \theta \cos ^{2} \Phi \sin ^{2} \Phi\right)$

$P_{13}^{\prime}=P_{11}\left(\cos ^{2} \theta \cos ^{4} \Phi \sin ^{2} \theta+\cos ^{2} \theta \sin ^{2} \theta \sin ^{4} \Phi+\right.$ $\left.\sin ^{2} \theta \cos ^{2} \theta\right)+P_{12}\left(\cos ^{2} \theta \sin ^{2} \Phi \sin ^{2} \theta \cos ^{2} \Phi+\right.$ $\cos ^{4} \theta \cos ^{2} \Phi+\cos ^{2} \theta \sin ^{2} \Phi \sin ^{2} \theta \cos ^{2} \Phi+$ $\left.\cos ^{4} \theta \sin ^{2} \Phi+\sin ^{4} \theta \cos ^{2} \Phi+\sin ^{4} \theta \sin ^{2} \Phi\right)+P_{44}$ $\left(\cos ^{2} \theta \cos ^{2} \Phi \sin ^{2} \Phi \sin ^{2} \theta+\cos ^{2} \theta \sin ^{2} \Phi \sin ^{2} \theta-\right.$ $\left.\cos ^{2} \theta \cos ^{2} \Phi \sin ^{2} \theta\right)$

$P_{44}^{\prime}=P_{1212}^{\prime}=P_{11}\left(\cos ^{2} \theta \cos ^{2} \Phi \sin ^{2} \Phi+\cos ^{2} \theta \sin ^{2} \Phi\right.$ $\left.\cos ^{2} \Phi\right)+P_{12}\left(-2 \cos ^{2} \theta \cos ^{2} \Phi \sin ^{2} \Phi-2 \cos ^{2} \theta\right.$ $\left.\cos ^{2} \Phi \sin ^{2} \theta\right)+P_{44}\left(\cos ^{2} \theta \cos ^{4} \phi+\sin ^{2} \theta \sin ^{2} \Phi\right)$

$P_{44}^{\prime}=P_{2323}^{\prime}=P_{11}\left(\sin ^{2} \Phi \sin ^{2} \theta \cos ^{2} \Phi+\cos ^{2} \Phi \sin ^{2} \theta\right.$ $\left.\sin ^{2} \Phi\right)+P_{12}\left(-2 \sin ^{2} \Phi \cos ^{2} \Phi \sin ^{2} \theta\right)+P_{44}\left(\sin ^{4} \Phi\right.$ $\left.\sin ^{2} \theta+\cos ^{2} \Phi \cos ^{2} \theta\right)$

$P_{44}^{\prime}=P_{3131}^{\prime}=P_{11}\left(\cos ^{2} \theta \cos ^{4} \phi \sin ^{2} \theta+\cos ^{2} \theta\right.$ $\left.\sin ^{4} \phi \sin ^{2} \theta+\sin ^{2} \theta \cos ^{2} \theta\right)+P_{12}\left(2 \cos ^{2} \theta \cos ^{2} \Phi\right.$ $\sin ^{2} \Phi \sin ^{2} \theta-2 \cos ^{2} \theta \cos ^{2} \Phi \sin ^{2} \theta-2 \cos ^{2} \theta \sin ^{2} \theta$ $\left.\sin ^{2} \Phi\right)+P_{44}\left(\cos ^{2} \theta \sin ^{2} \Phi \sin ^{2} \theta \cos ^{2} \Phi+\sin ^{4} \theta\right.$ $\left.\sin ^{2} \Phi+\cos ^{4} \theta \cos ^{2} \Phi\right)$

According to Scanning Infrared Polariscope method, the photoelastic constants need to be evaluated in the form of $P_{11}^{\prime}-P_{12}^{\prime}$ and $P_{44}^{\prime}$. Therefore, after employing some mathematical manipulation, we have derived the photoelastic constants in the form given below; 
$P_{11}^{\prime}-P_{12}^{\prime}=\left(P_{11}-P_{12}\right)\left[1-3 \cos ^{2} \theta \sin ^{2} \theta-3 \cos ^{4} \theta\right.$

$\left.\cos ^{2} \Phi \sin ^{2} \Phi\right]+\frac{3}{2} P_{44}\left[\cos ^{4} \theta \cos ^{2} \Phi \sin ^{2} \Phi+\right.$ $\left.\cos ^{2} \theta \sin ^{2} \theta\right]$

$P_{44}^{\prime}=\frac{1}{3}\left(P_{11}-P_{12}\right)\left[2 \cos ^{2} \Phi \sin ^{2} \Phi+2 \cos ^{2} \theta \sin ^{2} \theta\right.$

$\left.-2 \cos ^{2} \theta \cos ^{2} \Phi \sin ^{2} \Phi \sin ^{2} \theta\right]+\frac{1}{3} P_{44}\left[\cos ^{2} \theta\right.$

$\cos ^{4} \phi+\sin ^{2} \theta \sin ^{2} \Phi+\sin ^{4} \phi \sin ^{2} \theta+\cos ^{2} \Phi$

$\cos ^{2} \theta+\sin ^{2} \theta \cos ^{2} \Phi \cos ^{2} \theta \sin ^{2} \Phi+\sin ^{4} \theta \sin ^{2} \Phi+$ $\left.\cos ^{4} \theta \cos ^{2} \Phi\right]$

\subsection{Mathematical Analysis FOR System MODEL2}

For the second system model, to express the angle $\phi$ in terms of $\alpha$ from the two equations (6) and (8), after employing some mathematical manipulation we get

$$
\phi=\cos ^{-1} \sqrt{\frac{2 \cos ^{2} \alpha}{1+\cos ^{2} \alpha}}
$$

We can also write $\theta$ in terms of $\alpha$ by equation (6)

$$
\theta=\tan ^{-1}\left[\sin \left(\cos ^{-1} \sqrt{\frac{2 \cos ^{2} \alpha}{1+\cos ^{2} \alpha}}\right)\right]
$$

Substituting equations (20) and (21) into equations (18) and (19) the photoelastic constants can be represented as

$$
\begin{aligned}
& P_{11}^{\prime}-P_{12}^{\prime}=\left(P_{11}-P_{12}\right)\left[1-3 \cos ^{2}\left\{\tan ^{-1}\left[\sin \left(\cos ^{-1} \beta\right)\right]\right\}\right. \\
& \sin ^{2}\left\{\tan ^{-1}\left[\sin \left(\cos ^{-1} \beta\right)\right]\right\}-3 \cos ^{4}\left\{\tan ^{-1}\left[\sin \left(\cos ^{-1} \beta\right)\right]\right\} \\
& \cos ^{2}\left\{\cos ^{-1} \beta\left\{\sin ^{2}\left\{\cos ^{-1} \beta\right\}\right]+\frac{3}{2} P{ }_{44}\left[\cos ^{4}\left\{\tan ^{-1}\left[\sin \left(\cos ^{-1} \beta\right)\right]\right\}\right.\right. \\
& \cos ^{2}\left\{\operatorname { c o s } ^ { - 1 } \beta \left\{\sin ^{2}\left\{\cos ^{-1} \beta\right\}+\cos ^{2}\left\{\tan ^{-1}\left[\sin \left(\cos ^{-1} \beta\right)\right]\right\}\right.\right. \\
& \left.\sin ^{2}\left\{\tan ^{-1}\left[\sin \left(\cos ^{-1} \beta\right)\right]\right\}\right]
\end{aligned}
$$

And,

$$
\begin{aligned}
& P_{44}^{\prime}=\frac{1}{3}\left(P_{11}-P_{12}\right)\left[2 \cos ^{2}\left\{\cos ^{-1} \beta\right\} \sin ^{2}\left\{\cos ^{-1} \beta\right\}+2 \cos ^{2}\right. \\
& \left\{\tan ^{-1}\left[\sin \left(\cos ^{-1} \beta\right)\right]\right\} \sin ^{2}\left\{\tan ^{-1}\left[\sin \left(\cos ^{-1} \beta\right)\right]-2 \cos ^{2}\right. \\
& \left\{\tan ^{-1}\left[\sin \left(\cos ^{-1} \beta\right)\right]\right\}-2 \cos ^{2}\left\{\tan ^{-1}\left[\sin \left(\cos ^{-1} \beta\right)\right]\right\} \cos ^{2}\left(\cos ^{-1} \beta\right)
\end{aligned}
$$

$$
\begin{aligned}
& \left.\sin ^{2}\left\{\tan ^{-1}\left[\sin \left(\cos ^{-1} \beta\right)\right]\right\} \sin ^{2}\left(\cos ^{-1} \beta\right)\right]+\frac{1}{3} P 44\left[\operatorname { c o s } ^ { 2 } \left\{\tan ^{-1}\right.\right. \\
& \left\{\left[\sin \left(\cos ^{-1} \beta\right)\right]\right\} \cos ^{4}\left\{\cos ^{-1} \beta\right\}+\sin ^{2}\left\{\tan ^{-1}\left[\sin \left(\cos ^{-1} \beta\right)\right]\right\} \\
& \sin ^{2}\left\{\cos ^{-1} \beta\right\}+\sin ^{2}\left\{\tan ^{-1}\left[\sin \left(\cos ^{-1} \beta\right)\right]\right\} \sin ^{4}\left\{\cos ^{-1} \beta\right\} \\
& +\cos ^{2}\left\{\tan ^{-1}\left[\sin \left(\cos ^{-1} \beta\right)\right]\right\} \cos ^{2}\left\{\cos ^{-1} \beta\right\}+\sin ^{2}\left\{\tan ^{-1}\right. \\
& \left\{\left[\sin \left(\cos ^{-1} \beta\right)\right]\right\} \cos ^{2}\left\{\cos ^{-1} \beta\right\} \cos ^{2}\left\{\tan ^{-1}\left\{\left[\sin ^{-1}\left(\cos ^{-1} \beta\right)\right]\right\}\right. \\
& \sin ^{2}\left\{\cos ^{-1} \beta\right\}+\sin ^{4}\left\{\tan ^{-1}\left[\sin \left(\cos ^{-1} \beta\right)\right]\left\{\left[\sin \left(\cos ^{-1} \beta\right)\right]\right\}\right. \\
& \left.+\cos ^{4}\left\{\tan ^{-1}\left[\sin \left(\cos ^{-1} \beta\right)\right]\right\} \cos ^{2}\left\{\cos ^{-1} \beta\right\}\right](23)
\end{aligned}
$$

Where, $\beta=\sqrt{\frac{2 \cos ^{2} \alpha}{1+\cos ^{2} \alpha}}$

\subsection{RESULTS AND DISCUSSIONS}

We have determined some numerical values of the unknown photoelastic constants for different crystal orientations with the known values of photoelastic constant along with a graphical representation. Here photoelastic constants are calculated using equations (18), (19), (22) and (23) for Si crystal as an example.

\subsection{DETERMINATION OF PHOTO ELASTIC CONSTANT FROM (100) TO (110)}

By putting $\theta$ equal to zero and varying $\phi$ from $0^{\circ}$ to $45^{\circ}$ in equation (18) and (19), values of $P_{11}^{\prime}$ $P_{12}^{\prime}$ and $P_{44}^{\prime}$ in XY plane are calculated. 

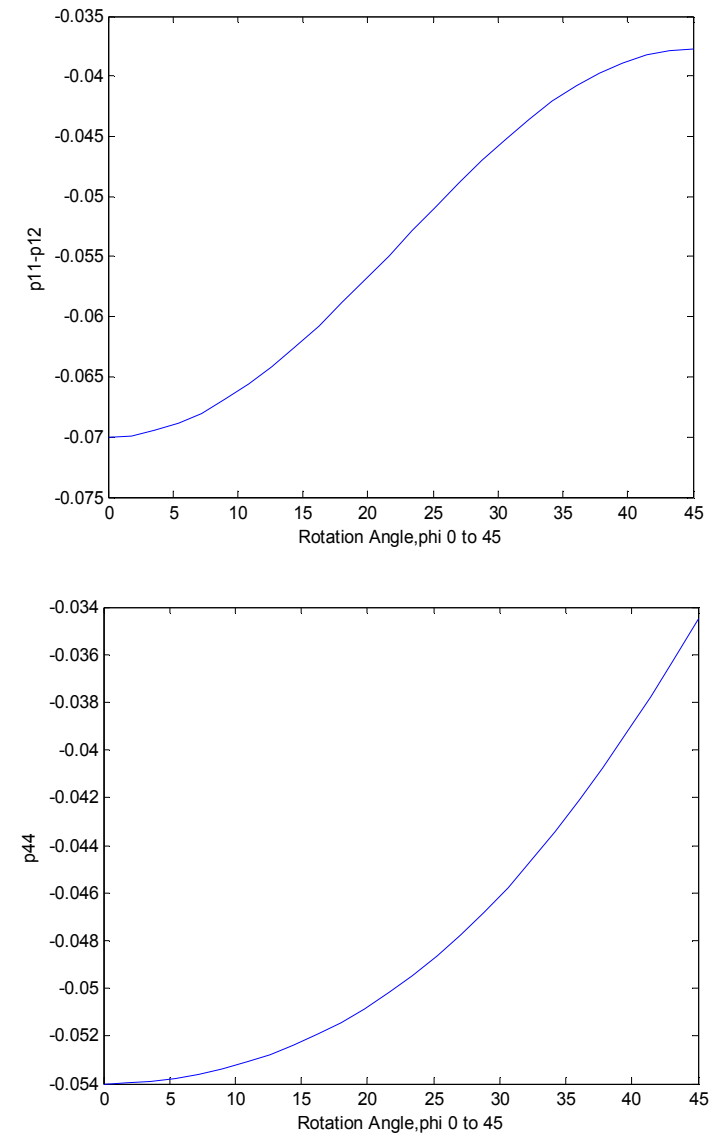

Fig.3: Variation of $P_{11}^{\prime}-P_{12}^{\prime}$ and $P_{44}^{\prime}$ with respect to different rotation angles

4.2 DETERMINATION OF PHOTO ELASTIC CONSTANT FROM (110) TO (001)

Here we have calculated the values of $P_{11}^{\prime} P_{12}^{\prime}$ and $P_{44}^{\prime}$ in XZ plane by putting $\phi$ equal to 45 and varying $\theta$ from $0^{\circ}$ to $90^{\circ}$ in equations (18) and (19). A graphical representation is given below.
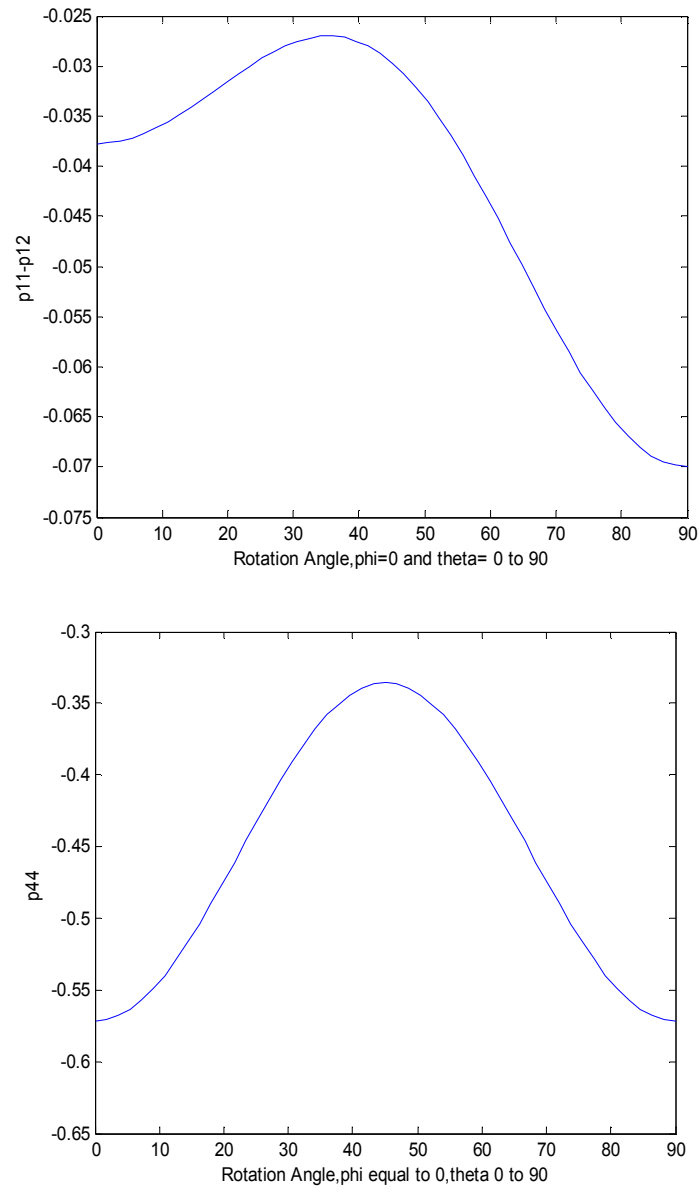

Fig 4: Variation of $P_{11}^{\prime}-P_{12}^{\prime}$ and $P_{44}^{\prime}$ with respect to different rotation angles

4.3 Determination of Photo Elastic CONSTANT From (100) TO (001)

In this case by putting $\phi$ equal to 0 and varing $\theta$ from $0^{\circ}$ to $90^{\circ}$ in equations (18) and (19), the values of $P_{11_{-}}^{\prime} P_{12}^{\prime}$ and $P_{44}^{\prime}$ are calculated. A graphical representation is given below. 

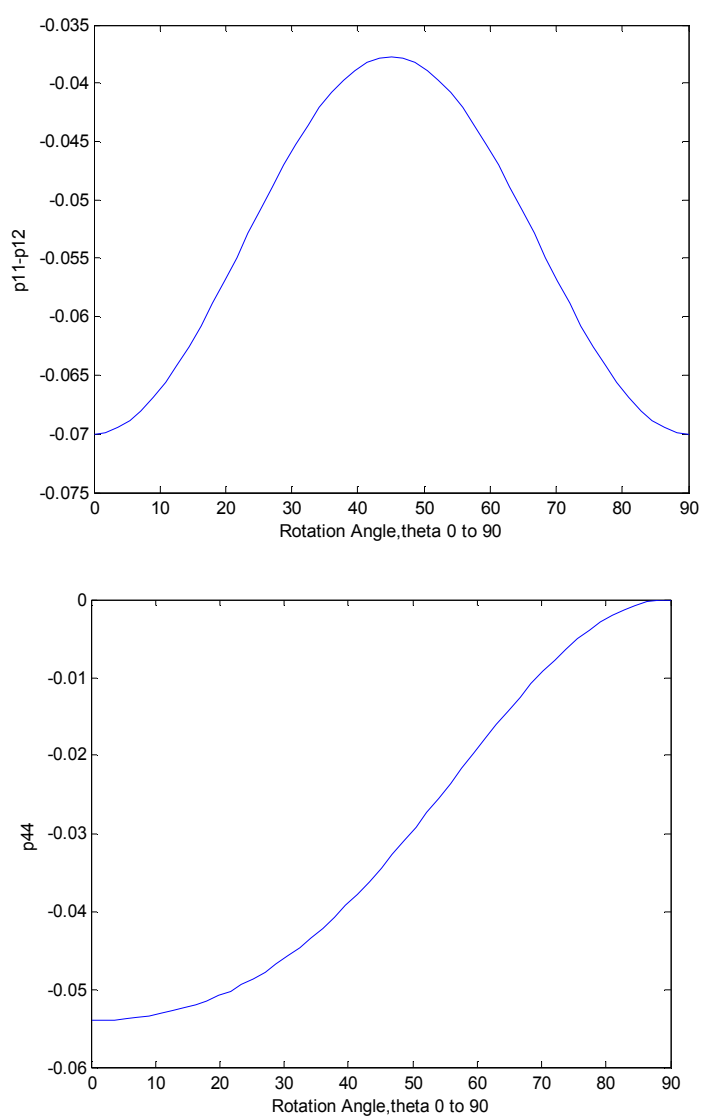

Fig. 5: Variation of $P_{11}^{\prime}-P_{12}^{\prime}$ and $P_{44}^{\prime}$ with respect to different rotation angles

\subsection{Determination of Photo Elastic CONSTANT From (100) TO (011)}

Here we have calculated the values of photoelastic constants by equations (22) and (23). By varying $\alpha$ from $0^{\circ}$ to $90^{\circ}$, the values of $P_{11}^{\prime} P_{12}^{\prime}$ and $P_{44}^{\prime}$ are calculated. A graphical representation is given below.

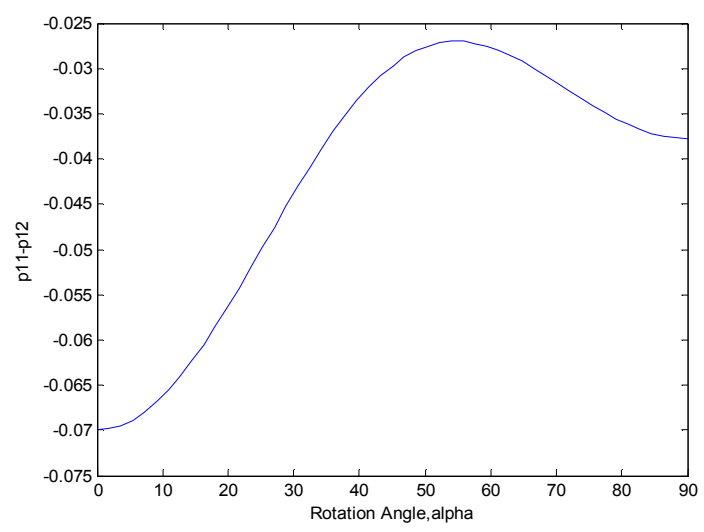

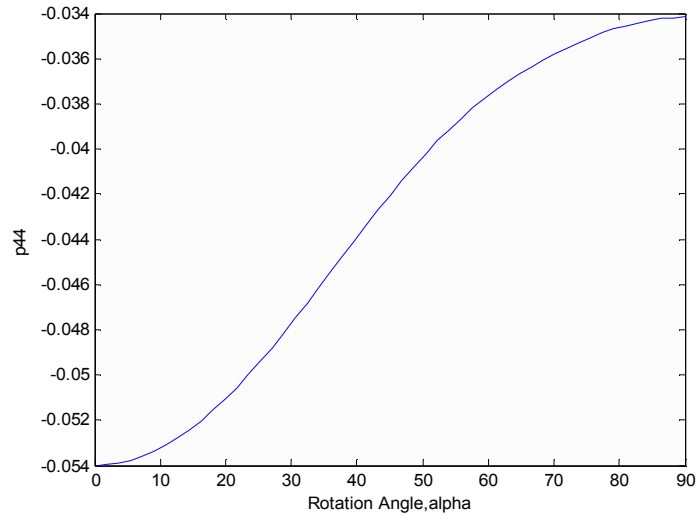

Fig. 6: Variation of $P_{11}^{\prime} P_{12}^{\prime}$ and $P_{44}^{\prime}$ with respect to different rotation angles

From the above figures, it is clear that the photoelastic constant $P_{44}^{\prime}$ and $P_{11}^{\prime}-P_{12}^{\prime}$ vary nonlinearly with the angle of rotation. The values of $P_{44}^{\prime}$ and $P_{11}^{\prime}-P_{12}^{\prime}$ give sinusoidal variation, exponential variation or harmonic variation with respect to rotation angles. This demonstrates that photoelastic constants, $P_{11}^{\prime}-P_{12}^{\prime}$ and $P_{44}^{\prime}$ are strongly dependent on crystal orientation. By putting the numerical values of photo elastic constants, $P_{11}^{\prime}-P_{12}^{\prime}$ and $P_{44}^{\prime}$ from the above figures in equation (1) and (2), we can characterize the components of strain with the help of Scanning Infrared Polariscope (SIRP) method.

\subsection{CONCLUSION}

In this work, we have derived mathematical formulations based on tensor rotation technique to determine the photoelastic constants in the form of $P_{11}^{\prime}-P_{12}^{\prime}$ and $P_{44}^{\prime}$ in arbitrary crystal orientations. Using these formulations, $P_{11}^{\prime}-P_{12}^{\prime}$ and $P_{44}^{\prime}$ can be determined in any particular crystal orientation by choosing the values of rotation angles. To characterize the strain induced in the polycrystalline material during the growth and cooling processes by means of Scanning Infrared Polariscope method it is very important to know the values of photoelastic constants corresponding to the crystal orientation. Though the model is showed for silicon material, it can also be applied for any material of Zincblende group. 


\section{REFERENCES}

[1] M. Yamada, Quantitative photoelastic characterization of residual strains in LEC-grown indium phosphide (100) wafers, Proc. Of $5^{\text {th }}$ Int. Conf. on InP and Related Materials, Paris France, IEEE Cat. \#93CH3276-3, pp. 69-72, (1993)

[2] Henderson R.H. \& E. Towe, Journal of applied physics 79 (4), 15 February 1996.

[3] "Euler's Rotation Theorem", http:// mathword.wolfram.com/Euler Angles.html

[4] T. S. Narasimhamutry, Photoelastic and ElectroOptic Properties of Crystals, Plenum press New York.

[5] Jun Chen, Bin Chen, Takashi Sekiguchi, masayuki Fukuzawa and Masayoki Yamada, Applied Physics Letters 93, 112105 (2008).

[6] Atsuko Niwa, Tsukuru Ohtoshi and Takao Kuroda, "Orientation Dependence of Optical Properties in Long Wavelength Electrical Electronic and Engineering Department Strained Quantum-Well Lasers", IEEE journal of selected topics in quantum electronics, Vol. 1, No. 2, June 1995. 